\title{
Resolution in Periodontal Inflammation- A Review
}

\author{
Dr. Nitin Kudyar ${ }^{1,}$ Dr. Vadiraj.S ${ }^{2}$ \\ ${ }^{1}$ (Registrar, Department of Periodontics, Indira Gandhi Government Dental College and hospital, Jammu, \\ India) \\ ${ }^{2}$ (Reader, Department of Periodontics, P.D.U Dental College and Hospital, Solapur, India)
}

\begin{abstract}
When inflammation is initiated, goal of host response is to quickly return to homeostasis through rapid elimination of invading leucocytes. Resolution of inflammation is an active process. Cells and events are stimulated to respond, which is quite different from blocking pro-inflammatory pathways.

The Harward school medical team identified a new class of fats, resolvins, which control inflammation by stopping the migration of inflammatory cells. Eicosapentaenoic acid acts as a precursor for resolvins which are produced by omega-3 fatty acids.

Discovery of resolvins and their use in an inflammatory disease model have provided insight into the determinants of susceptibility of periodontics.

Prevents repair, helps regeneration.....

Key Words: LIPOXIN, RESOLUTION, RESOLVINS,
\end{abstract}

\section{Introduction}

Periodontitis is a chronic multifactorial disease. Various aspects of innate and acquired immunity play role in the pathogenesis of periodontitis. When an inflammatory event is initiated by bacteria, viruses or injury, the goal of the host response is to quickly return to homeostasis through rapid elimination of invading leukocytes. When the process is slow, as in chronic diseases, there will be destruction of cellular matrix and will lead to scarring. A similar process occurs in case of chronic periodontitis, where scarring refers to the arresting of bone destruction without gain of gingival attachment or bone height ${ }^{1}$.

Since years, anti-inflammatory agents are used for prevention and management of inflammatory diseases. However anti-inflammation is not the same as resolution. Once the inflammatory response is initiated, a continuous cascade of events takes place. During this series of events, the body attempts to eliminate invaders through proinflammatory actions of cells and their products. A basic theory of modern medicine is that upon neutralization of the invader, inflammation resolves due to catabolism of 'proinflammatory' mediators.

The process of resolution of inflammation, similar to 'proinflammatory' mechanisms, utilizes cells and various messenger molecules generated by cells to provide 'stop signals' that lead to shut-down and clearance of inflammatory cells. Thus, inflammation includes both proinflammatory and resolving mechanisms inherent to the body where the host attempts to confine and/or eliminate the invaders and when accomplished, actively resolves the response to limit damage to self. Hence, the body has the capacity to actively control inflammation.

\section{Resolution And Repair}

'Pro-resolving' mediators are readily generated in host tissues. These factors limit leukocyte channelization directly into the inflamed site, reverse the cardinal signs of inflammation such as vasodilation and vascular permeability, and coordinate the clearance of exhausted leukocytes, exudates, and fibrin; eventually leading to the restoration of function. All of these inflammation-resolving processes limit and prevent tissue injury and further progression of acute inflammation into chronic inflammation.

If there is a failure of the host in its ability to eliminate the injury in acute phase, inflammation proceeds to a chronic phase and results in varying degrees of tissue injury. When tissue injury is mild and confined, necrotic cells will be replaced by new cells via regeneration. If tissue damage is extensive, the process of healing is repair (scarring). When repair takes place, fibrin is not cleared rapidly and efficiently and granulation tissue is formed from surrounding tissue compartments. Later phases of repair involve fibroblastmediated collagen deposition, disappearance of vascular tissues and replacement of these areas by avascular and fibrotic scar tissue.

Thus, in the context of resolution of inflammation, 'acute resolution' leads to regeneration, whereas 'chronic resolution' results in repair. These terms are applicable to periodontal tissue healing. In periodontal disease pathogenesis, similar to other forms of host-mediated tissue injury, such as rheumatoid arthritis and asthma, chronic resolution also leads to ongoing tissue damage through continuous and recurring episodes of acute inflammation. Therefore, the initiation and resolution of inflammation are two parallel phases in chronic 
inflammation and such pathologies should be defined as 'continuous' inflammatory diseases rather than independent stages.

Untill recently it was thought that resolution of inflammation is due to gradual decrease in molecules such as prostaglandins, leukotrienes and interleukins. Now its clear that resolution of inflammation is affected by a new class of lipid molecules that emerge in late inflammation (fig.-1) and are known as lipoxins and resolvins ${ }^{2,3}$. These molecules bind to distinct receptors on the cells which alter the function of the cell leading to resolution and healing. Due to the presence of these lipid molecules in resolution phase it is clear that the resolution is an active process ${ }^{4}$ rather than passive as it was earlier thought. The term resolvins (resolution phase interaction products) was first introduced to emphasize that these are endogenous products possessing anti inflammatory properties ${ }^{5}$.( Fig.-1)

\section{Endogenous Production Of Resolving Molecules}

Lipoxins are generated late in the inflammation when a second lipoxegenase interacts with lipoxegenase produced earlier by other cell ${ }^{3}$ and it will bind to specific receptors on neutrophils leading to resolution. These lipoxins prevent chemotaxis of neutrophils leading to apoptosis which further acts as a chemotactic agent for mononuclear phagocytes. In the presence of lipoxins mononuclear phagocytes have nondestructive (nonphlogistic) function ${ }^{6,7}$. Thus periodontal inflammation leading to tissue destruction is due to lack of resolution and proinflammatory stimuli.

Using the same metabolic pathways, omega- 3 fatty acids in the diet are the substrate for the formation of resolvins and protectins ${ }^{8}$. Dietary polyunsaturated fatty acids can substitute arachidonic acid of host origin producing resolvins and protectins having same properties as lipoxins ${ }^{8}$. Fish oil is rich in $\omega-3$ fatty acids and the consumption of fish oil increases the ratio of $\omega-3$ fatty acids to $\omega-6$ fatty acids in the cell membrane. This is in concordance with studies revealing that experimental animals fed with fish-oil-enriched diet produce less arachidonic-acid-derived inflammatory mediators ${ }^{9,10,11}$.

The main receptors for resolvins are the chemR-23 receptors expressed on macrophages and leukotriene-4-receptor-1 on neutrophils ${ }^{12}$. This new family of chemical mediator- resolvins and protectins are defined by their potent bioactivity and novel chemical structures. Since the precursors of resolvins and protectins are derived from omega-3 polyunsaturated fatty acids, there is a clear link that omega-3 supplementation of diet reduces inflammatory disease ${ }^{13,14}$. The protectins were so named because of their protective actions against stroke ${ }^{11}$, animal models of alzheimer's disease ${ }^{16}$ and other diseases of neural systems ${ }^{17}$. Interestingly when aspirin is taken along with omega-3 fatty acids in our diet, a new type of resolvins are formed which are more stable and longer acting $g^{5}$. There is new data to suggest that part of action of aspirin is due to derivatization of resolvins and lipoxins ${ }^{8}$.

\section{Role Of Aspirin In Resolution}

Aspirin (ASA) is unique among current therapies because it acetylates cyclooxygenase (COX)-2 enabling the biosynthesis of resolvin-containing precursors of endogenous antiinflammatory mediators. Human COX-2 converts docosahexanoic acid (DHA) to 13-hydroxy-DHA (HDHA) that switches with ASA to 17RHDHA which is also proved to be a major route in hypoxic endothelial cells. Human neutrophils transform COX-2-ASA-derived 17R-hydroxy-DHA into two sets of novel di and tri hydroxy products; one initiates via oxygenation at carbon 7 and the other at carbon 4 . These compounds inhibit microglial cell cytokine expression and in vivo dermal inflammation and peritonitis at nanogram (ng) doses, reducing 40-80\% leukocytic exudates. These results indicate that exudate, vascular, leukocyte and neural cells treated with aspirin convert DHA to novel 17R-hydroxy series of docosanoids that are potent regulators of resolution. These biosynthetic pathways of proresolution lipid mediators utilize omega-3 DHA and eicosapentanoic acid(EPA) during multicellular events in resolution to produce a family of protective compounds, i.e., resolvins and protectins, that enhance proresolution status ${ }^{18}$.(Fig.2)

\section{Application Of Resolvins In Prevention And/Or Management Of Periodontitis}

Prevention of P.gingivalis induced periodontitis by topical application of resolvins was evaluated in a 6 week experiment by Hasturk H.et $\mathrm{al}^{18}$. Teeth were ligatured at baseline and P.gingivalis was applied to the ligature $\left(10^{13}\right.$ P.gingivalis colonies in a methyl cellulose slurry) three times weekly to two groups of animals (New zealand white rabbit). One group received $5 u \underline{1}$ of a $1 u \underline{g} / \mathrm{ml}$ solution of resolvin E1 in ethanol (topically) and other group received ethanol alone (placebo). At the end of six week treatment period, animals were sacrificed and periodontal disease progression was quantified morphologically and histologically. Significant progression of periodontal disease, including bone and attachment loss, was observed in the placebo group. Resolvin E1 application prevented the onset and progression of periodontal disease in experiment group.

In a similar study by Hasturk $\mathrm{H}$. et $\mathrm{al}^{19}$ periodontal disease induced by P.gingivalis was established as above for 6- weeks. After 6- weeks, application of P.gingivalis was stopped, and animals (New Zealand, white 
rabbit) were divided into three groups. The first group was sacrificed at 6 -weeks to determine baseline of periodontal destruction. The second group was given treatment of resolvins E1 thrice a week in topical form as same regime as above for additional 6 weeks. Third group was given placebo treatment same as above. At the end of additional 6 -weeks, the animals were sacrificed, and periodontal destruction was quantified. Progression of disease was significant with deepening of pockets and additional loss of crestal bone. Tooth mobility also increased significantly. In the resolvin E1 treated group, there was complete elimination of inflammation; pocket depth was reduced to normal and soft tissue returned to preligature architecture level.

In another study by Hasturk $\mathrm{H}^{20}$, periodontitis was induced with ligature and P. gingivalis in 39 animals for a 6 -weeks period. Five animals were randomly selected and sacrificed at 6 -weeks to confirm the establishment of periodontal disease. The remaining 34 animals were divided into 5 treatment groups: $1=$ nontreatment $\{5$ animals $\} ; \quad 2=$ vehicle(ethanol) $\{5$ animals $\} ; \quad 3=$ LTB4(leukotrienes) $\quad\{5$ animals $\} ; \quad 4=$ PGE2(prostaglandins) $\{5$ animals $\} ; 5=\operatorname{RvE} 1$ (resolvins) $\{14$ animals $\}$. Topical application of all treatments was performed every-other-day for 6- weeks. Morphometric, radiographic and histologic evaluations were performed. Bone loss was measured directly on defleshed jaws. Histologic sections were stained with hematoxylin-eosin and tartrate-resistant acid phosphatase (TRAP) for descriptive histology and osteoclastic activity. In addition, Masson's trichrome staining was performed to detect the newly generated bone. The result of this study was that the application of $P$. gingivalis together with ligatures led to the development of periodontitis. Significant disease progression was detected in groups with no treatment, vehicle, LTB4 and PGE2 treatments. Conversely, local RvE1 application reversed the inflammatory changes including inflammatory cell infiltration and osteoclastic activity induced by $P$. gingivalis.

\section{Conclusion}

A large body of recent work suggests that the resolvins, associated with inflammation, are the molecules responsible for the resolution of inflammation. These molecules have been demonstrated to be important in a variety of disease processes, and their therapeutic potential has been identified in a variety of model systems. It has been demonstrated that resolution of inflammation in periodontitis through resolvinmediated pathways offers potential for the prevention and perhaps treatment of periodontal lesions. Future studies need to focus on the applicability of resolvin therapies in humans for the prevention and treatment of the periodontal diseases.

In the light of following facts, there is insufficient evidence to support the role of pro resolution lipid mediators in the management of periodontal disease:-

- Periodontal disease was initiated in experimental animals by P.gingivalis. Periodontal disease is considered to be a multifactorial disease where different organisms populating plaque in organized manner and lead to tissue destruction. If P.gingivalis would have been the sole etiologic agent, periodontal vaccine would have been developed a long ago.

- The duration allowed for the disease to develop in experimental animals was not sufficient to call it a chronic disease. The results obtained in these experiments may or may not resemble those in chronic periodontitis cases in humans which take years to develop.

Multiple multicentric longitudinal interventional studies in different population groups will be required to strongly prove the application of resolvins/lipoxins in resolution of inflammation in patients of periodontitis.

\section{References}

[1] Cotren RS, Kumar V, Collins T. Cellular Pathology I: Cell injury and cell death. Philadelphia: Saunders 1999; 1-29.

[2] Sehran CN, Brain SD, Gilroy DW, Haslett C, O’Neill LA, Perritti M, Rossi AG, Wallace JL. Resolution of inflammation: state of art, definitions and terms. FASEB 2007; 21:325-332.

[3] Sehran CN. A search of endogenous mechanisms of anti inflammation uncovers novel chemical mediators: missing links to resolution. Histochem Cell Biology, 122, 2004, 305-321.

[4] Sehran CN. Lipoxin biosynthesis and its impact in inflammatory and vascular events. Biochim Biophys Acta, 1212, 1994, $1212: 1-25$.

[5] Serhan CN, Hong S, Gronert K, Colgan SP, Devchand PR, Mirick G, Moussignac RL. Resolvins: a family of bioactive products of omega-3 fatty acid transformation circuits initiated by Aspirin treatment that counter proinflammation signal. J. Exp Me, 196, 2002 :1025-1037.

[6] Bannerberg GL, Chiang N, Ariel M, Tjonahen E, Gotlinger KH, Hongs, Sehran CN. Molecular circuits of resolution: formation and actions of resolvins and protectins. J Immunology, 174, 2005,4345-4355.

[7] Pouliot M, Clish CB, Petasis NA, Van Dyke TE, Sehran CN. Lipoxin A analogues inhibit leukocyte recruitment to Porphyromonas gingivalis. A role for cyclooxygenase-2 and lipoxins in periodontal disease. Biochemistry, 39, 2000,4761-4768.

[8] Sehran CN, Clish CB, Brannan J, Colgan SP, Chiang N, Grovert K. Novel functional sets of lipid derived mediators with anti inflammatory actions generated from omega-3 fatty acids via cyclooxygenase 2- nonsteroidal anti inflammatory drugs and transcellular processing. J. Exp. Med., 192, 2000, 1197-1204.

[9] Rosenstein Elliot D., Kushner Laura J., Kraner Neil, Kazandjian Gregory. Pilot study of dietary fatty acid supplementation in treatment of adult periodontitis. Prostaglandins, leukotrienes and essential fatty acids (march), 68:3, 2003 213-218.

[10] Saynur Vardar et al. Therapeutic versus prophylactic plus therapeutic administration of omega-3 fatty acids on endotoxin induced periodontitis in rats. J Periodontol 75(12), 2004; 1640-1646.

[11] Kesavalu L. et al. Omega-3 fatty acid effect on alveolar bone loss in rats. J Dent Res;.85(7) 2006,648-652. 
[12] Arita M, Ohira T, SunYP, Elangovan S, Chiang N, Sehran CN. Resolvin $E_{1}$ selectively interacts with leukotriene B 4 receptor BLT and chem. R-23 to regulate inflammation. J. Immunology; 178, 2007, 3912-3917.

[13] De Caterina R, Endres S, Kristensen SD, Schmidt EB, Editors. n-3 fatty acids and vascular disease. London: springer- Verlag; 1993:1-191.

[14] Jain A, Batista E, Serhan C, Stahl G, Van Dyke TE. A role of periodontitis in the progression of lipid deposition in an animal model. Infect Immun, 71, 2003, 6012-6018.

[15] Marchesethi VL, Hong S, Lukiw WJ, Hua Tian X, Gronert K, Musto A, Hardy M, Gimenez JM, Chiang N, Serhan CN, Bazan NG. Novel docosanoids inhibit brain ischemia/ reperfusion/ mediated leukocyte infiltration and proinflammatory gene expression. J.Bio.Chem, 278, 2003, 43807-43817.

[16] Lukiw WJ, Cin JG, Marcheselli VL, Bodker M, Botkjaer A, Gotlinger K, Serhan CN, Bazan NG. A role for docosahexaenoic acidderived Neutroprotectin $\mathrm{D}_{1}$ in neural cell survival and Alzheimer's disease. J. Clin. Invest, 115, 2005, 2774-2783.

[17] Mukherjee PK, Marcheselli VL, Serhan CN, Bazan NG. Neutroprotectin $D_{1}$ : A docosahexaenoic acid- derived docosatriene protects human retinal pigment epithelial cells from oxidative stress. Proc Natl Acad Sci USA , 101, 2004, 8491-8496.

[18] Hasturk H, Kantarci A, Ohira T, Arita M, Ebrahim N, Chirag N, Petasis N A, Levy B D, Serhan C N, Van Dyke T E. RvE 1 protects from local inflammation and osteoclast mediated bone destruction in periodontitis. FASEB; 20, 2006, 401-403.

[19] Hasturk H, Kantarei A, Goguer- Surmenian E, Blackwood A, Andy C, Petasis N, Serhan C, Van Dyke T. Resolvin E 1 regulates inflammation at the cellular and tissue level and restores tissue homeostasis in vivo. J.Immuno, 179, 2007,7021-7029.

[20] Hasturk H. et al. Resolution of inflammation and bone regeneration in periodontal disease, IADR/AADR/CADR, (March), 2007, 2124.

Fig.-1(Pathway of emergence of lipid molecules in inflammation ${ }^{2,3}$ )

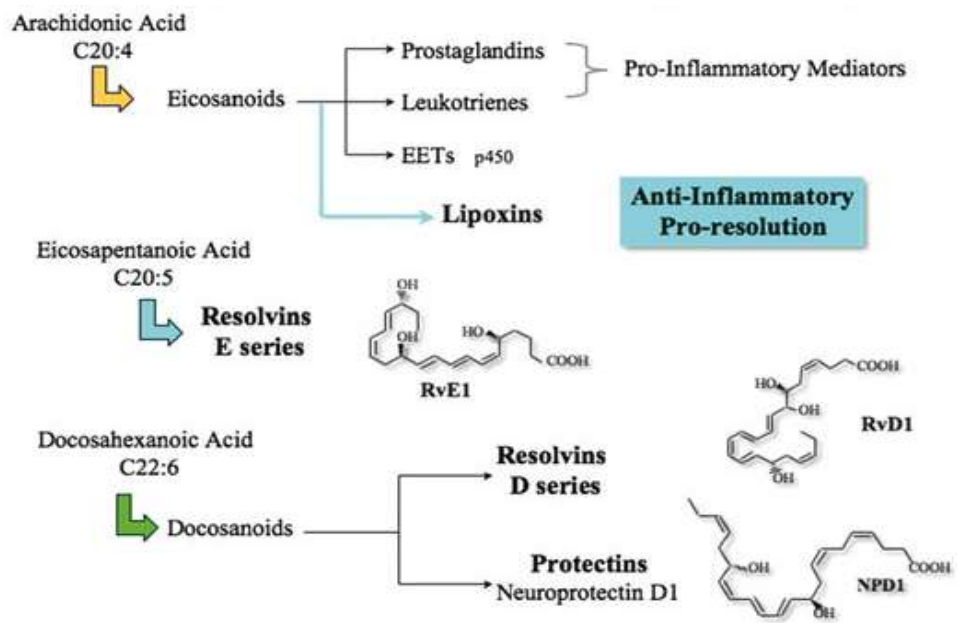

Fig.-2(Role of aspirin in resolution of inflammation ${ }^{18}$ ) The Protective (Pro-resolution) Lipid Mediators
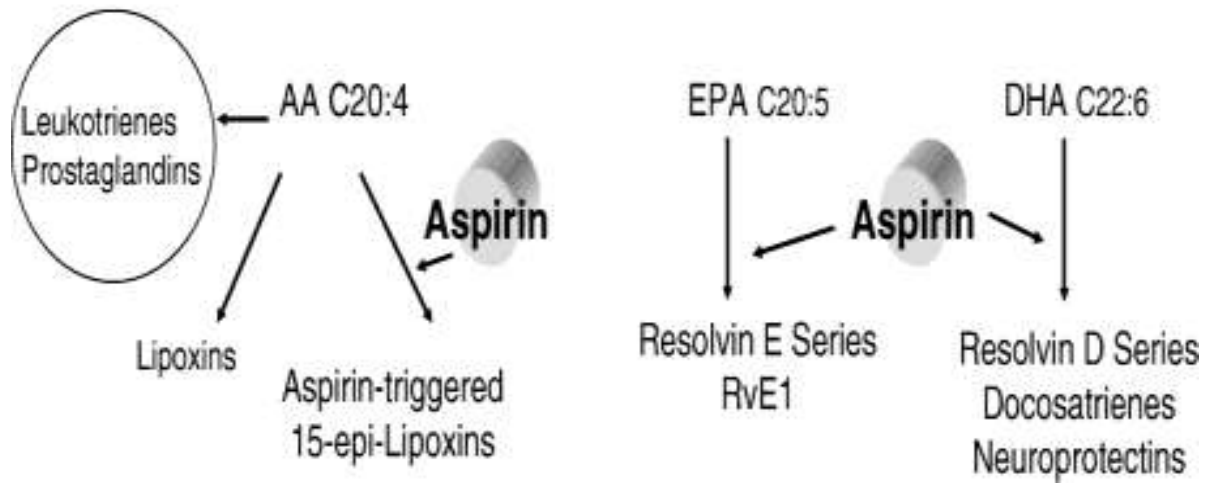